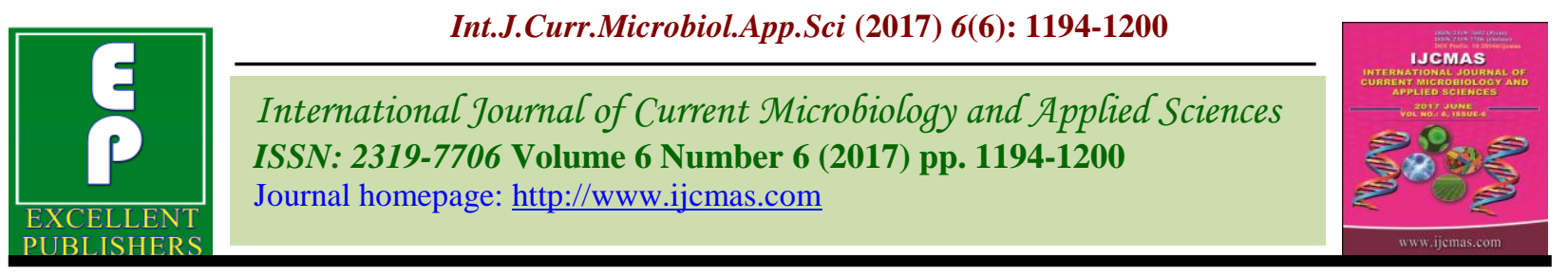

Review Article

https://doi.org/10.20546/ijcmas.2017.606.138

\title{
Inflamosomes as Activated Molecular Platform for Engagement of Innate Immune Defences
}

\author{
Shubhangi Warke, Sumedha Bobade*, D.R. Kalorey and V.C. Ingle \\ Nagpur Veterinary College, Maharashtra Animal and Fishery Sciences University, Nagpur, India \\ *Corresponding author
}

\begin{tabular}{|c|c|}
\hline & A B S T R A C T \\
\hline & \multirow{6}{*}{$\begin{array}{l}\text { Traditionally, innate immunity is first line of defence. These cells use } \\
\text { multiprotein complexes called inflammasomes to produce proteins known } \\
\text { as cytokines. The innate immune system plays a crucial role in the rapid } \\
\text { recognition and elimination of invading microbes. The inflammasomes are } \\
\text { large multiprotein complexes scaffolded by cytosolic pattern recognition } \\
\text { receptors (PRRs) that form an important part of the innate immune system. } \\
\text { In this review focus on relevant aspects concerning critical role of } \\
\text { inflamosomes in mediating host defence, emerging links between the } \\
\text { inflammasome and pyroptosis and autophagy, inflammasomes as design } \\
\text { effective, safe adjuvants in the future has been discussed. }\end{array}$} \\
\hline Keywords & \\
\hline DAMP, & \\
\hline $\begin{array}{l}\text { NLRC, NOD, } \\
\text { PPR, TLR. }\end{array}$ & \\
\hline Article Info & \\
\hline $\begin{array}{l}\text { Accepted: } \\
\text { 19 May } 2017 \\
\text { Available Online: } \\
\text { 10 June } 2017\end{array}$ & \\
\hline
\end{tabular}

\section{Introduction}

The term "inflammasome" was coined by Jurg Tschopp and colleagues at the University of Lausanne in Switzerland to describe a caspase activating complex that processes pro-IL1b (35kd) to mature IL1b (17kd form). Inflammasomes are multimeric complexes formed in response to a variety of physiological and pathogenic stimuli. Inflammasome activation is an essential component of the innate immune response and is critical for the clearance of pathogens or damaged cells (Sharma, and Kanneganti, 2016).

The innate immune system plays a primary role in the rapid recognition and elimination of invading microorganisms, through different processes such as phagocytosis and the induction of inflammation. Inflammation is characterized by activation of innate immune cells and production of proinflammatory cytokines IL- $1 \alpha$, IL- $1 \beta$, and $\mathrm{TNF} \alpha$, large multiprotein complexes that sense intracellular danger signals via NODlike receptors (NLR) (Szabo and Csak, 2012). Inflammasomes are multimolecular complexes that assemble into a platform for the activation of proinflammatory caspase 1 . These includes the Nod like receptor (NLR) proteins NLRP1, NLRP3, NLRC4, NLRP6 and Naip5, as well as the DNA sensing complex of AIM2 (Nature Immunology, 2012). 


\section{Pattern-recognition receptors (PRRs)}

PRRs recognize pathogen associated molecular patterns (PAMPs), and damage associated molecular patterns (DAMPs). There are multiple families of PRRs including the toll like receptors (TLRs), the $\mathrm{C}$ type lectins, the retinoid acid inducible gene (RIG) I receptors and the NLRs.

The cytoplasmic NLRs share structural homology with plant $\mathrm{R}$ proteins that serve to recognize plant pathogens (Jones and Dangl, 2006).

Toll like receptors (TLRs) are membrane bound pattern recognition receptors that recognize certain extracellular phagocytized pathogen associated molecular patterns (PAMPs) and danger associated molecular patterns (DAMPs). Nod like receptors represent a second line of defense against pathogens (Fernandez et al., 2014).

\section{NLR family}

A subset of PRRs, belonging to the nucleotide binding oligomerization domain (NOD) like receptor (NLR) families, detects viral and bacterial pathogens in the cytosol of host cells and induces the assembly of a multiprotein signaling platform called the inflammasome (Liu and Sun, 2012). To date, 22 NLRs have been identified in humans, and 34 have been identified in mice (Shaw et al., 2010).

The NLR family is further subdivided into four groups based on the amino terminus motif, which include NLRA with an acidic transactivation domain, NLRP with a pyrin domain (PYD), and NLRC containing a caspase recruitment domain (CARD), NLRB members with a baculoviral inhibitory repeat (BIR) like domain and NLRX with a nonhomologous amino terminal (Ting et al., 2008).

\section{Schematic representation of inflammasome} complexes (Dunne, 2011)

Inflammasome activation leads to the generation of active caspase 1 , which in turn cleaves the pro-form of IL- $1 \beta$ into a mature and active form. Unrestrained ligandindependent inflammasome activation or recognition of host-derived ligands leads to autoinflammatory and inflammatory disease respectively, whereas recognition of pathogen-derived molecules is required for infection clearance.

\section{Prototypes of inflammasomes}

\section{NLRP1 inflammasome}

NLRP1 (NACHT, LRR, and PYD domainscontaining protein 1), the first inflammasome described, can directly interact with caspase1 through its C-terminal CARD domain, and in humans, the presence of ASC enhances the activity of the complex (Schroder and Tschopp, 2010). Murine NLRP1 is unable to bind to ASC because it lacks a functional PYD domain. NLRP1 is activated by the muramyl dipeptide (MDP) and the Bacillus anthracis lethal toxin (Szabo and Csak, 2012). Humans possess one NLRP1 gene, while duplication events that occurred after the bifurcation of rodents and primates gave rise to 3 murine variants; Nlrp1a, Nlrp1b, and Nlrp1c, human NLRP1 has an N-terminal PYD motif, while its murine orthologs lack this motif.

\section{NLR3}

NLRP3, are involved in the response to pathogens. IL1b is the original 'endogenous pyrogen. NLRP3 seems to be unique in its promiscuous ability to be activated by a wide array of unrelated stimuli, including microbe or host derived molecules and even totally inorganic entities such as silica, asbestos and 
most famously, alum (Nature Immunology, 2012).

\section{NLRC4}

NLRC4 is an exemplar of the protective role of inflammasomes. The messy nature of pyroptosis is also an effective means of disseminating the inflammatory response and alerting the host to the presence of pathogens (Nature Immunology, 2012). NLRC4/IPAF (NLR-family CARD domain containing protein 4) inflammasome is activated by the flagellin of Gram-negative and Gram-positive bacteria or the type III secretion system (T3SS) of Gram-negative bacteria.

\section{NLRP6}

NLRP6 is uncharacteristic by virtue of the unusual ligand it recognizes and its observed downstream effects. NLRP6 identified as a negative regulator of the mitogen-activated protein kinase and NF- $\kappa B$ pathway in macrophages infected with Listeria monocytogenes, Escherichia coli, and Salmonella (Anand et al., 2012).

\section{NLRP12}

NLRP12 was initially characterized as an inhibitor of noncanonical NF- $\mathrm{BB}$ signaling (Lich et al., 2007). It was also involved in caspase-1 activation in response to Yersinia pestis and Plasmodium infection (Vladimer $e t$ al., 2012; Ataide et al., 2014). The role of NLRP12 in NF- $\mathrm{KB}-$ mediated signaling makes it an important regulator of immune response after Salmonella infection and during colorectal cancer (Zaki et al., 2014)

\section{AIM 2 inflammasome}

AIM 2 (absent in melanoma 2) is a cytosolic dsDNA sensing inflammasome activated by bacterial, viral, and mammalian host DNA to trigger caspase-1 activation (Nakahira et al.,
2011). AIM2 can directly bind to its ligand and may contribute to the pathogenesis of autoimmune diseases by recognizing the mammalian DNA (Rathinam et al., 2010).

\section{Inflammasome Activation}

NLRP3 inflammasome assembly occurs following activation of the NLPR3 protein. Several NLRP3 inflammasome activators have been identified, and these comprise viruses (including influenza virus, adenovirus), bacteria (including Staphylococcus aureus, Listeria monocytogenes, Shigella flexneri), fungi, bacterial pore forming toxins, ATP, crystalline particulates (including alum, asbestos, silica, uric acid), chemical irritants and UVB (Wilson and cassel, 2010).

\section{Inhibitors of Inflamosomes}

\section{Pyroptosis and Appoptosis}

Inflammasome assembly leads to the activation of the proinflammatory caspase. Consequently, an inflammatory immune response is mounted along with a programmed cell death, called pyroptosis (Jamilloux et al., 2014). NLRC4 is an exemplar of the protective role of inflammasomes. IL1b is the original 'endogenous pyrogen', Inflamosomes and autophagy

The autophagy and inflammasome pathways are ancient innate immune mechanisms for controlling invading pathogens that are linked by mutual regulation. As a cytosolic pathogen recognition receptor (PRR) complex, the inflammasome both induces and is induced by autophagy through direct interactions with autophagy proteins or through the effects of secondary molecules, such as mitochondrial reactive oxygen species and mitochondrial DNA (Rodgers et al., 2014). 


\section{Inflamosomes and adjuvanticity}

Adjuvants are materials that enhance the immune response to an antigen. The NALP3 inflammasome has been associated with several autoinflammatory conditions including gout. The NALP3 inflammasome is a crucial element in the adjuvant effect of aluminum and can direct a humoral adaptive immune response. Many of the inflammasome activators have adjuvant properties; these include MDP (muramyl dipeptide), MSU (monosodium urate), alum, silica dust. Alum defines particle materials based on aluminium salt precipitates that are the most widely used adjuvants in human vaccines. Alum activates a Th2-biased immunity with elevated Th2-dependent antibody isotypes IgG1 and IgE, both MSU and alum depend on an intact inflammasome including NALP3, ASC, and caspase-1 to trigger a Th2-biased response (Martinon et al., 2009).

Schematic representation of inflammasome complexes (Dunne, 2011)

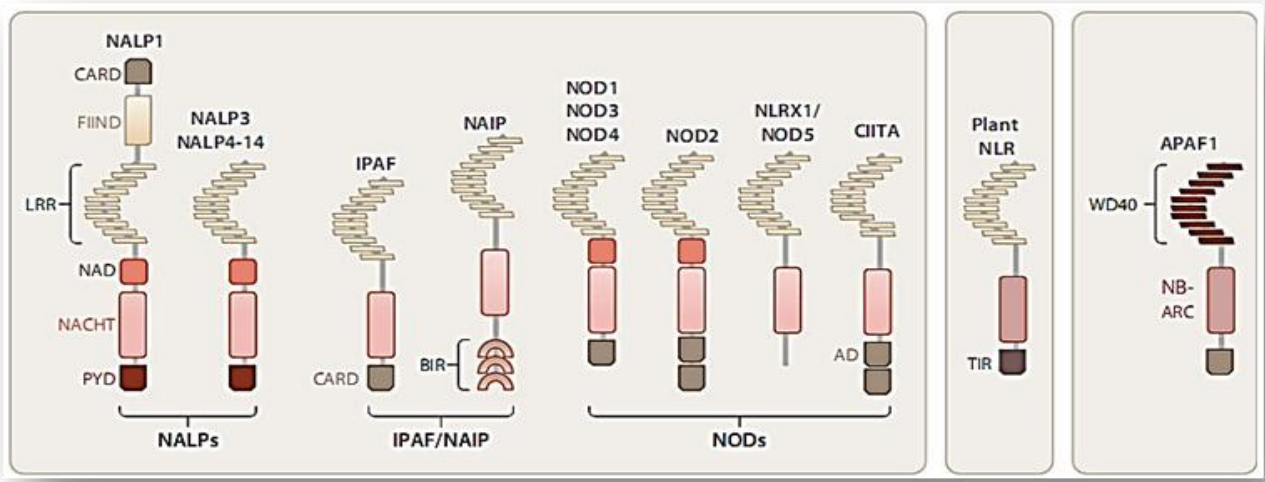

Activation of the NLRP1B inflammasome by anthrax lethal toxin

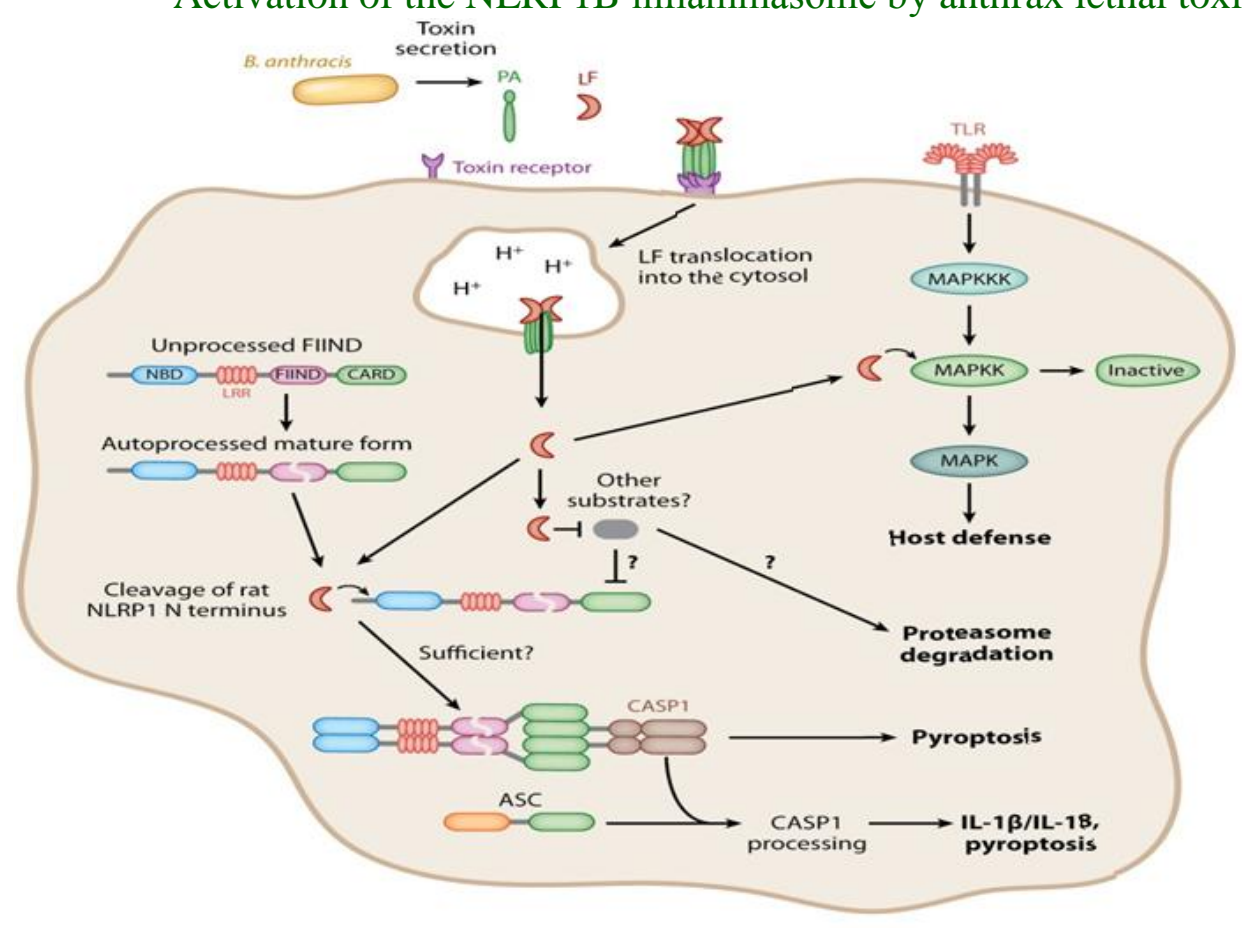




\section{Inflamosomes and disorders}

The discovery that NLRP3 (NLR-related protein 3) can recognize host-derived particulate matter such as uric acid and cholesterol crystals has led to this inflammasome being implicated in a number of inflammatory diseases, including gout, atherosclerosis and Type 2 diabetes. In addition, aberrant NLRP3 activation has also been observed in a number of heritable disorders now referred to as cryopyrinopathies (Dunne, 2011).

Atherosclerosis has also been associated with chronic inflammation. Accumulation of immune cells and cytokine production are hallmarks of atherosclerotic plaques (Hansson, 2005). IL-1 $\beta$ is one of the proinflammatory cytokines involved in the pathogenesis of metabolic disorders (Davis et al., 2011). Inflammasomes have been linked to a variety of autoinflammatory and autoimmune diseases, including neurodegenerative diseases (multiple sclerosis, Alzheimer's disease and Parkinson's disease) and metabolic disorders (atherosclerosis, type 2 diabetes and obesity) (Strowig et al., 2012).

\section{Inflamosomes and carcinogens}

The inhibition of inflammasomes or neutralization of their products, mainly interleukin $1 \beta$ (IL1 $\beta$ ) and IL18, has profound effects on carcinogenesis and tumor progression. Thus, inflammasomes are promising therapeutic targets in cancer related clinical conditions (Zitvogel et al., 2012). The clinical importance of inflammasomes reaches beyond infectious disease, as dysregulated inflammasome activity is associated with numerous hereditary and acquired inflammatory disorders (Broz and Dixit, 2016).
A major challenge in NLR and inflammasome research is that current knowledge of NLR signaling pathways and disease function is highly fragmented. The long-term aim of the "NLR and inflammasome signaling" research unit is to gain insight into the role of NLRs and inflammasomes in human disease. Translation of newly gained knowledge will contribute to the development of innovative diagnostics and therapeutic approaches for autoimmune and infectious diseases. Recently, inflammasome function has been implicated in more common human conditions, such as gout, type II diabetes and cancer. This raises the possibility that anti-IL1 therapeutics may have broader applications and may be utilized across diverse disease states that are linked insidiously through unwanted or heightened inflammasome activity. In addition to removal of damaged cells, inflammasomes are also involved in cell repair, metabolism, and proliferation. Various molecules believed to be involved in the maintenance of cellular homeostasis have been demonstrated to act as critical regulators of inflammasome function and vice versa.

\section{References}

Anand, P.K., R.K. Malireddi, J.R. Lukens, P. Vogel, J. Bertin, M. Lamkanfi, and T.D. Kanneganti. 2012. NLRP6 negatively regulates innate immunity and host defence against bacterial pathogens. Nature, 488: 389-393. http://dx.doi.org /10.1038 /nature11250.

Aninflammatory assemblage. 2012. Nature Immunol., 13: 320.doi:10.1038/ni.2268.

Ataide, M.A., W.A. Andrade, D.S. Zamboni, D. Wang, M.C. Souza, B.S. Franklin, S. Elian, F.S. Martins, D. Pereira, G. Reed, et al. 2014. Malaria-induced NLRP12/NLRP3-dependent caspase-1 activation mediates inflammation and hypersensitivity to bacterial super infection. PLoS Pathog., 10: e1003885. 
Published erratum appears in PLoS Pathog., 10: e1004258) http://dx.doi.org /10.1371 /journal.ppat.1003885

Broz, P. and Dixit, V.M. 2016. Inflammasomes: mechanism of assembly, regulation and signalling. Nature Rev. Immunol., 16: 407-420, doi:10.1038/nri.2016.58.

Davis, B.K., Wen, H., and. Ting, J.P. 2011. The Inflammasome NLRs in Immunity, Inflammation, and Associated Diseases. Annu. Rev. Immunol., 29: 707-735. doi:10.1146/annurev-immunol-031210101405 .

Dunne, A. 2011. Inflammasome activation: from inflammatory disease to infection. Biochem. Soc. Trans., 39: 669-673; doi: 10.1042/BST0390669.

Fernandez, M.V., Miller, E.A., Bhardwaj, N. 2014. Activation and Measurement of NLRP3 Inflammasome Activity Using IL1 $\beta$ in Human Monocyte derived Dendritic Cells. J. Vis. Exp., 87: e51284, doi: 10.3791/51284.

Hansson, G.K. 2005. Inflammation, atherosclerosis, and coronary artery disease. N. Engl. J. Med., 352: 1685-95.

Hornung, V., Latz, E. 2010. Intracellular DNA recognition. Nat. Rev. Immunol., 10: 123-130.

Jamilloux, Y., Sève, P., Henry, T. 2014. Inflammasomes in human diseases. Rev. Med. Int., 35(11): 73041. doi: 10.1016/j.revmed.2014.04.017.

Jones, J.D., and Dang, J.L. 2006. The plant immune system. Nature, 444: 323-329.

Lich, J.D., K.L. Williams, C.B. Moore, J.C. Arthur, B.K. Davis, D.J. Taxman, and J.P. Ting. 2007. Monarch-1 suppresses non-canonical NF-kappaB activation and p52-dependent chemokine expression in monocytes. J. Immunol., 178: $\quad 1256-1260 . \quad$ http://dx.doi.org /10.4049 /jimmunol.178.3.1256.

Liu, A.N., and Sun, T.Y. 2012. Regulation of NOD like receptors and inflammasome during the inflammation, Sheng Li Xue Bao, 64(6):74150.

Martinon, F., Mayor, A., Tschopp, J. 2009. The inflammasomes: guardians of the body. Annu. Rev. Immunol., 27: 229265.

Moltke, J., N.J. Trinidad, M. Moayeri, A.F. Kintzer, S.B. Wang, N. Van Rooijen, C.R. Brown, B.A. Krantz, S.H. Leppla, K. Gronert, and R.E. Vance. 2012. Rapid induction of inflammatory lipid mediators by the inflammasome in vivo. Nature, 490: 107-111.

Moltke, J.V., Ayres, J.S., Kofoed, E.M., Chavarria-Smith, J., Russell, E.V. 2013. Recognition of Bacteria by Inflammasomes. Annu. Rev. Immunol., 31: 73-106.

Nakahira, K., Haspel, J.A., Rathinam, V.A., Lee, S.J., Dolinay, T., Lam, H.C., et al. 2011. Autophagy proteins regulate innate immune responses by inhibiting the release of mitochondrial DNA mediated by the NALP3 inflammasome. Nat. Immunol., 12: 222-230.

Rathinam, V.A., Jiang, Z., Waggoner, S.N., Sharma, S., Cole, L.E., Waggoner, L., Vanaja, S.K., Monks, B.G., Ganesan, S., Latz, E., Hornung, V., Vogel, S.N., Szomolanyi Tsuda, E., and Fitzgerald, K.A. 2010. The AIM2 inflammasome is essential for host defense against cytosolic bacteria and DNA viruses. Nat. Immunol., 11: 395-402.

Rodgers, M.A., Bowman, J.W., Liang, Q., Jung, J.U. 2014. Regulation where autophagy intersects the inflammasome. Antioxid. Redox. Signal, 20(3): 495506. doi: 10.1089/ars.2013.5347.

Schroder, K., and Tschopp, J. 2010. The Inflam masomes, Cell, 140: 821-832.

Sharma, D., and Kanneganti, T. 2016. The cell biology of inflammasomes: Mechanisms of inflammasome activation and regulation. J. Cell Biol., 213(6): 617. 
Shaw, M.H., Franchi, L., Coban, C., Ishii, K. J., Akira, S., Horii, T., Rodriguez, A., and Nunez, G. 2010. Experimental cerebral malaria progresses independently of the Nlrp3 inflammasome. Eur. J. Immunol., 40: 764-769.

Strowig, T., Henao-Mejia, J., Elinav, E. and Flavell, R. 2012. Inflammasomes in health and disease. Nature, 481: 278286.

Szabo, G.and Csak, T. 2012. Inflammasomes in liver diseases. J. Hepatol., 57: 642654.

Ting, J.P., Lovering, R.C., Alnemri, E.S., Bertin, J., Boss, J.M., et al. 2008. The NLR gene family: a standard nomenclature. Immunity, 28: 285-287.
Vladimer, G.I., D. Weng, S.W. Paquette, S.K. Vanaja, V.A. Rathinam, M.H. Aune, J.E. Conlon, J.J. Burbage, M.K. Proulx, Q. Liu, et al. 2012. The NLRP12 inflammasome recognizes Yersinia pestis. Immunity, 37: 96-107.

Zaki, M.H., S.M. Man, P. Vogel, M. Lamkanfi, and T.D. Kanneganti. 2014. Salmonella exploits NLRP12-dependent innate immune signaling to suppress host defenses during infection. Proc. Natl. Acad. Sci. USA, 111: 385-390. http://dx.doi.org/10.1073/pnas.1317643 111.

Zitvogel, L., Kepp, O., Galluzzi, L., and Kroemer, G. 2012. Inflammasomes in carcinogenesis and anticancer immune responses. Nature Immunol., 13: 343351, doi:10.1038/ni.2224.

\section{How to cite this article:}

Shubhangi Warke, Sumedha Bobade, D.R. Kalorey and Ingle, V.C. 2017. Inflamosomes as Activated Molecular Platform for Engagement of Innate Immune Defences. Int.J.Curr.Microbiol.App.Sci. 6(6): 1194-1200. doi: https://doi.org/10.20546/ijcmas.2017.606.138 